\title{
An Overview of Error Management Climate
}

\author{
Linqing Li \\ Management School, Jinan University, Guangzhou, China \\ Email: 13526594715@163.com
}

Received 9 March 2016; accepted 25 April 2016; published 28 April 2016

Copyright (C) 2016 by authors and Scientific Research Publishing Inc.

This work is licensed under the Creative Commons Attribution International License (CC BY). http://creativecommons.org/licenses/by/4.0/

(c) (i) Open Access

\begin{abstract}
As a kind of organizational management environment, error management climate has great influence on employee behavior and organizational performance. This paper combines the latest research, summarizes the connotation and the mechanism of error management climate, and summarizes its structural dimensions. At last, the paper introduces the error management climate factors and the related empirical studies.
\end{abstract}

\section{Keywords}

Error Management Climate, Error Management Mechanism, Related Research

\section{The Origin and Development of Error Management Climate}

In organizations, errors may always exist. For example, it cannot finish the task before deadline, forgetting to send a message in time, hiring the wrong person, and so on. For a long time, people think that making mistakes are very bad things; it will bring serious loss to the organization; in the organization, people should try to avoid the occurrence of errors, and do a good job in the prevention of errors. However, enterprise practice proves that due to the information acquisition for people to making decision is limited; the error cannot completely prevent (Zhao, 2006). Wherever it may produce, so researchers become to treat the error more scientifically (Dyck, 2005), shift attention to how to reduce the harm as much as possible after the error occurred, and ultimately learn from errors, and promote the organizational goals on. Frese et al. (1991) proposed the concept of error management, different from error prevention; error management is to provide a learning opportunity for the organization members, so that they know how to adjust and change their behavior. The difference between error prevention and error management is: error prevention produces before the error occurred, and is to try to reduce the number of errors; error management occurs after the error, treats error as the cause and tries to control the false negative effects (Keith \& Frese, 2005). Currently most enterprises will adopt error prevention measures, however, error prevention can only achieve the purpose of control error, pure error prevention cannot teach people how to deal with errors and learn from the mistake of strategy (Huber, 1991). In contrast, error management teaches people how to learn from mistakes. Error is not good in itself, but when it happens, it will be very 
useful for the organization to learn from the mistakes and improve the work flow. Error management can guarantee that the error is found and reported in the first time, while eliminate the negative impact caused by the error and learn from it, which is needed by the modern enterprise especially Internet company's management.

\section{The Definition of the Concept of Error Management Climate}

\subsection{Distinguish of Error Related Concepts}

Error management climate is part of positive organizational culture, and error is defined as the accident deviation behavior from goals or expectations. Keith \& Frese (2011) distinguished several relative concepts: firstly, the distinction between errors and violation is that errors refer to unintentional deviation from the original plans, objectives, rules or standards, probably (but not necessarily) will have negative consequences, such as damage, stress, and depression; and the violation refers to intentionally deviate from the rules or standards in order to achieve a certain purpose. Secondly, the difference between mistakes and failures: failure is a negative result, it may be caused by errors, but not all the mistakes will lead to failure. If the error can be found timely and correctly, it may not lead to failure. Thirdly, the difference between mistakes and low performance: errors and low performance may lead to the same results such as cannot complete the task in time, and the difference between the two is that errors will make people have a feeling: I can do better. Dyck (2005) defined the error management: error management does not mean to eliminate all errors, but to go after the error handling and its consequences. The error management can also be applied to the organization level, namely error management culture. Organizational error management culture refers to the organization and organizational procedures, error related practices and attitudes, and is the way the method of error handling. The latest research of error management culture covers into a more comprehensive concept of error management climate. Cigularov, Chen, \& Rosecrance (2010) considered that error management climate (EMC) refers to a kind of organization atmosphere, which promoted "error communicating and sharing, helping each other in the error environment, the exploration and analysis of the error, reducing negative influence and recovering quickly from the mistakes in” after an error occurred.

\subsection{Distinguish of Error Management Climate Related Concepts}

Error management culture refers to organizational procedures, error related practices and attitudes, and the method of error handling of organizations. Error management culture emphasizes a set of guidelines, value conception system that is shared in the organization, which is outward and visible; and error management climate contains not only outward and visible the value concept system, but also an internalized atmosphere. Error prevention appears before the error occurred, and is possible to eliminate the error; error management is in order to curb the negative influence, to promote the positive effects of false error.

\section{Error Management Mechanism}

The main purpose of error management is handling errors quickly and efficiently, reducing the loss and harm caused by the error, learning from mistakes and the possible future error prevention. The study found that error management mechanism lies in the following three points: 1) timely monitoring, in-depth analysis and open communication after error occurred; 2) effectively handling errors and negative consequences; 3) looking for the opportunity to reverse from the mistakes and find valuable information to learn (Cigularov, Chen, \& Rosecrance, 2010).

\section{The Structure and Measurement of Error Management Climate}

Based on the reviews of the literature, the dimensions of error management climate are summarized as below. Study on error management climate dimensions of foreign scholars are mainly in the following aspects: Frese et al. (1991) found that the error can be divided into two dimensions: emotional mood and action dimensions. Emotional dimension contains the risk of error, error prediction, error pressure and error concealment; action dimension contains error learning, error thinking, error competence and error pressure; Rybowiak et al. (1999) studied individual orientation for error, and formed the individual orientation error attitude questionnaire which contains eight dimensions: error ability, error learning, error communication, error thinking, risk undertaking, 
error prediction, error competence and error pressure; Dyck (2005) divided error management culture into two dimensions: error management culture and error avoidance culture. Error management culture includes four dimensions: error mastery, error communication, error undertaking and error antipathy. Gold et al. (2013) proposed error management climate blame orientation and error management climate open orientation: blame orientation does not allow employees to make an error, and open orientation will treat error as a normal thing, allowing the employees to make mistakes. Based on Van Dack et al. (2005) theory, the error management climate has been verified by Chinese scholars such as Tsai (2009). He considered that error management climate included four dimensions: error control, error communication, error bear and error antipathy.

\section{The Relevant Empirical Study of Error Management Climate}

The empirical research on error management climate can be divided into two aspects: the influence factors and the effect of the results (Yin, 2012). Firstly, research shows that transformational leaders are good at promoting staff to explore, trial and error, and so that have positive effects on error management climate; the laissez-faire leadership always disregard organization's objectives, ignores employees' ask, and is not conducive to the construction of organizational error management climate. Research on performance appraisal orientation and error management climate indicates that performance appraisal target to evaluate the alignment, and is not conducive to the formation of error management climate. Development orientation of performance appraisal tends to reduce the punishment of error or failure on employee, which can promote the formation of error management climate.

Secondly, the research about the effect of error management climate focuses on organizational performance, organizational or employee learning, reducing error of adverse effects, secondary error prevention, promoting employee innovative behavior, and encouraging employees to exploration, trial and error, improving the quality of products and services and so on (Dyck, 2005).

The study of Gronewold (2013) in a professional service organization found that error management climate will affect employee's voluntary error reporting intentions: high error management climate can bring higher willingness to report self false, with this relationship regulated by the type of error. Compared to the operational errors, the concept errors are more able to strengthen the relationship between error management climate and the willingness to report self false. At the same time, it is found that the error management climate can promote the generation of employee's moral behavior, especially among the junior staff. With empirical study Dyck (2005) found that: the organization's error management culture and organizational goals, objective economic performance is significantly related. At the same time, error management culture has a positive correlation with organizational performance, while error pressure is negatively related to organizational performance. Cigularov, Chen, \& Rosecrance (2010) conducted a survey of 235 American construction associations to investigate the influence of the Contractor's error management climate and the safety of the workers on the safety behavior and injury. The results show that the error management climate and security communication have significant positive effects on the reduction of workers' injuries. Good error management climate and security communication are helpful to improve the safety of the construction industry. Cai Yuqiao's (2009) investigation of 152 Taiwan business people from various industries has found that: forward error management culture has positive influence on organizational learning and organizational performance and organizational learning plays an intermediary role between error management culture and organizational performance. Wang (2000)'s study of the 168 subjects from 50 companies found that the error mastery dimension of error management climate has significantly positive correlation with organizational performance; the error stress dimension and organizational performance was significantly negatively correlated. Zhu \& Pei (2014) investigated 227 employees and found that error management culture has a significant positive impact on employee Innovative behavior, and psychological empowerment plays an intermediary role between the two and innovation efficacy can positively regulate the relationship between psychological empowerment and employee’s innovative behavior. Ma (2015) surveyed 562 employees of 105 companies and found that: open oriented error management climate can promote knowledge socialization and externalization; blame oriented error management climate can promote the combination of knowledge and internalization. Open oriented error management climate can promote employee's ability of exploratory innovation; blame oriented error management climate can promote employee’s ability of utilization innovation.

\section{Prospect}

Although there has been a lot of research on error management climate at home and abroad, most of the scholars 
still focus on the theoretical research, which is rarely related to the empirical study of the error management climate. Based on this, the future research can consider how to apply the error management climate to the management practice, and explore how it interacts with the employee's emotion and attitude. In addition, because most of the research on the error management climate is positive, the future direction can also take into account the negative error management climate.

\section{References}

Cigularov, K. P., Chen, P. Y., \& Rosecrance, J. (2010). The Effects of Error Management Climate and Safety Communication on Safety: A Multi-Level Study. Accident Analysis \& Prevention, 42, 1498-1506. http://dx.doi.org/10.1016/j.aap.2010.01.003

Frese, M., Brodbeck, F., Heinbokel, T. et al. (1991). Errors in Training Computer Skills: On the Positive Function of Errors. Human-Computer Interaction, 6, 77-93. http://dx.doi.org/10.1207/s15327051hci0601_3

Gold, A., Gronewold, U., \& Salterio, S. E. (2013). Error Management in Audit Firms: Error Climate, Type, and Originator. The Accounting Review, 89, 303-330. http://dx.doi.org/10.2308/accr-50592

Gronewold, U., Gold, A., \& Salterio, S. E. (2013). Reporting Self-Made Errors: The Impact of Organizational Error-Management Climate and Error Type. Journal of Business Ethics, 117, 189-208. http://dx.doi.org/10.1007/s10551-012-1500-6

Huber, G. P. (1991) Organizational Learning: The Contributing Processes and the Literatures. Organization Science, 2, 88115. http://dx.doi.org/10.1287/orsc.2.1.88

Keith, N., \& Frese, M. (2005). Self-Regulation in Error Management Training: Emotion Control and Metacognition as Mediators of Performance Effects. Journal of Applied Psychology, 90, 677. http://dx.doi.org/10.1037/0021-9010.90.4.677

Keith, N., \& Frese, M. (2011). Enhancing Firm Performance and Innovativeness through Error Management Culture. Handbook of Organizational Culture and Climate, 9, 137-157.

Ma, L. (2015) Error Management Climate, Knowledge Transformation and Study of the Relationship between the Ability of the Staff. Chongqing University of Posts and Telecommunications.

Rybowiak, V., Garst, H., Frese, M. et al. (1999). Error Orientation Questionnaire (EOQ): Reliability, Validity, and Different Language Equivalence. Journal of Organizational Behavior, 20.

Van Dyck, C., Frese, M., Baer, M. et al. (2005). Organizational Error Management Culture and Its Impact on Performance: A Two-Study Replication. Journal of Applied Psychology, 90, 1228. http://dx.doi.org/10.1037/0021-9010.90.6.1228

Wang, C. H. (2000). Hong Self-Reliance. Study on the Relationship between Error Management Climate and Organizational Effectiveness. Journal of Zhejiang University: Humanities and Social Sciences Edition, 30, 114-119.

Tsai, Y.-C. (2009). The Relationship between Error Management Culture and Organizational Performance: The Mediating Effect of Organizational Learning.

Yin, R. F. (2012). Research on the Influence of Leadership Style on Management Culture and Organizational Innovation Performance. Leadership Science, 26, 43-45.

Zhao, B. (2006). Error Reporting in Organizations. Academy of Management Review, 31, 1012-1030. http://dx.doi.org/10.5465/AMR.2006.22528167

Zhu, Y. J., \& Pei, Y. (2014) Error Management Culture, Effect of Psychological Empowerment on Employee Innovative Behavior: Creative Self-Efficacy Moderating Effect. China Human Resources Development, 17, 23-29. 